\title{
CUERPOS EN EXPERIMENTACIÓN. SÍFILIS Y FÁRMACOPODER EN LA CiUdad de GuATEMALA \\ (1946-1948)
}

\author{
Human Experimentation. SyPhiLIS AND \\ pharmacopower in Guatemala City (1946-1948)
}

\section{Marco Chivalán Carrillo* \\ DOI: https://doi.org/10.31644/ED.V7.N2.2020.A05}

Resumen: Los experimentos practicados con cuerpos humanos, para la generación de un fármaco profiláctico post exposición a la sífilis, en la Ciudad de Guatemala entre 1946 y 1948 han sido condenados por la bioética. Sin embargo, esta práctica parece ser más compleja al analizarla como dispositivo de producción corporal desde el fármacopoder. Mediante un cruce de perspectivas epistemológicas, este estudio plantea que el uso de cuerpos "anómalos", en tal dispositivo, muestra una paradoja en la producción de lo "completamente humano". Y, para interferir tal producción, propone una alianza micropolítica de los "anómalos". Este trabajo pretende ser un aporte para una analítica más amplia del dispositivo de producción corporal en Guatemala. Las conclusiones arrojan la relevancia de profundizar y extender la comprensión de la problemática a través de diversos fondos de archivo.

Palabras claves: fármacopoder, sífilis, cuerpos, experimentación, Guatemala.

\footnotetext{
* Investigador k'iche' en ciencias sociales en el Área de Estudios sobre Imaginarios Sociales de la Asociación para el Avance de las Ciencias SocialesGuatemala. Es candidato a Doctor en Historia comparada, historia social, historia política e historia regional por la Universidad de Murcia, España. Líneas de investigación: : feminismos, procesos de producción de subjetividad, historia política del "cuerpo" e historia de la moral. Correo-e: markoich@gmail.com.

Fecha de recepción: 23/12/2019. Fecha de aceptación: 05/02/2020. Fecha de publicación: $31 / 07 / 2020$
}

(cc) BY-NC-ND Páginas 127 - 159 
Abstract: The experimentations on human bodies, to produce a prophylactic post-exposure drug for syphilis, which took place in Guatemala City (1946-1948), has been widely condemned by bioethical studies. However, this practice appears more complex when it is analyzed, for instance, as a "pharmacopower" device of corporeal production. In order to examine this complexity, this study combines epistemological perspectives to explore how the use of "anomalous" bodies, by this device, demonstrates a paradox in the production of the "complete human". And, to disrupt this production, this study proposes a micropolitical alliance with those considered "anomalous." Finally, the purpose of this study is to contribute to the understanding of the complexity of body production devices in Guatemala. The conclusions demonstrate the relevance of deepening and extending our understanding of the problem by drawing on diverse archival sources.

Keywords: pharmacopower, syphilis, human body, experimentation, Guatemala.

\section{Introducción}

Los cuerpos viles son los condenados a muerte, los convictos, los detenidos, los huérfanos, las prostitutas, los internados, los pacientes de hospital, los paraliticos, los esclavos, los colonizados, los moribundos que históricamente han servido como material experimental para la formación de la ciencia médica moderna. ${ }^{1}$ Grégoire Chamayou (2008)

Las construcciones de los limites de un organismo, el trabajo de los discursos de la inmunología, son poderosos mediadores de las experiencias de enfermedad y de muerte para los seres vivos industriales y postindustriales.

Donna Haraway (1995)

A través de este texto, propongo una analítica concerniente a los usos del cuerpo en el nada inocente aparato de experimentación humana. El corpus

\footnotetext{
1 "Les corps vils, ce sont les condamnés à mort, les bagnards, les détenus, les orphelines, les prostituées, les internés, les patients de l'hôpital, les paralytiques, les esclaves, les colonisés, les moribonds qui ont historiquement servi de matériel expérimental pour la constitution de la science médicale moderne" [traducción libre del autor] (Chamayou, 2008: 7).
} 
de análisis se ha circunscrito al material que registra los experimentos con humanos suscitados en Guatemala a finales de los cuarenta del siglo pasado. Este acontecimiento se refiere a la experimentación con seres humanos para la generación de un fármaco profiláctico post exposición a la sífilis. Tal experimento fue realizado bajo la dirección del Dr. John Cutler, médico estadounidense. El formato que toma este escrito combina el reporte de investigación sobre el caso y reflexiones teórica-filosóficas.

El interés que sostengo en esta narrativa sobre los cuerpos de experimentación es continuar problematizando las diversas tecnologías de producción corporal y de la subjetividad en un país de herencia colonial como Guatemala. La secuencia de este texto sigue este formato: en primer lugar, esbozo someramente los hechos de experimentación desarrollados de 1946 a 1948 bajo la dirección del Dr. Cutler. En segundo lugar, hilvano mis reflexiones en torno a la gestación de los cuerpos de experimentación. Las propuestas teóricas de las que nutro mi argumentación vienen principalmente de Michel Foucault (1990; 1995; 2005), Donna Haraway (1995; 1999; 2004a; 2004b; 2016), Achille Mbembe (2006), Teresa de Lauretis (2000) y Paul Beatriz Preciado (2008); y, para el caso de Latinoamérica, relaciono estas propuestas con los trabajos de Eugenia Bianchi $(2018 ; 2019)$ y Susana Murillo (2008; 2015a; 2015b; 2018). Una lectura cruzada entre estxs ${ }^{2}$ autores me permitió desarrollar una tecnología política de la experimentación con seres humanos en un cronotopo etnoespecíficamente situado como lo es "Guatemala a mediados del siglo XX". En tales técnicas, diagramo una lectura en la que la gestión de los cuerpos, producidos como figuras sifilíticas, responde a la necropolítica y a la fármacopolítica. Dicho de otro modo, la gestión técnica de la vida y de la muerte pasa por una gestión profiláctica del cuerpo y de la subjetividad.

En tercer lugar, bosquejo una reflexión final, la cual ofrece una lectura cruzada sobre los imaginarios coloniales y los estudios sobre animales. Esta reflexión la he circunscrito en un, apresuradamente, denominado indianimalia, siguiendo aproximaciones de los estudios queer animal. Al seguir

\footnotetext{
${ }^{2}$ El uso de la "x" responde al lenguaje inclusivo para corroer el binarismo normativo de género.
} 
las investigaciones de Grégoire Chamayou (2008; 2012), propongo mostrar cómo las prácticas científicas que él analiza cobran un estatuto empírico en los experimentos realizados en Guatemala, pero de otro modo. Finalmente, en el mismo apartado, delineo algunas ideas como manera de concluir -o, no- este texto. Para tal apartado no prometo una conclusión, sobre todo porque con ello propongo una posibilidad de problematizar el esbozo de esta hipótesis sobre el reciclaje de una tecnología política colonial en tanto que aparato de producción corporal con enormes efectos en la historia presente de Guatemala: La simbiosis humanobacteria parece ser el intensificador del cuerpo "longevo/ lozano/nacional/digno de vida”.

\section{El hecho}

La práctica de experimentación con seres humanos en Guatemala fue desarrollada de 1946 a 1948. Los archivos que la registraron fueron descubiertos por Susan Reverby (2011: 6-28), quien los halló accidentalmente cuando indagaba sobre los experimentos realizados en hombres negros: los Tuskegee Studies (2011: 6) -estudios desarrollados por el mismo Servicio de Salud Pública (PHS por sus siglas en inglés) de Estados Unidos en Tuskegee, Alabama. El caso guatemalteco salió a luz pública y fue por este que el primero de octubre de 2010 la Secretaria de Estado, Hillary Rodham Clinton, y la Secretaría del Departamento de Salud y Servicios Humanos (DHHS por sus siglas en inglés), Kathleen Sebelius, ofrecieron una disculpa formal al Estado de Guatemala por esta investigación (2011: 2) $)^{3}$. A este estudio lo calificaron, sin lugar a dudas, de "abominable", "no ético" y "condenable". Al revisar la prensa escrita y el trabajo de Susan Reverby sobre los archivos, se constata que el presidente Barack Obama telefoneó al que fungía como Presidente de Guatemala en el 2010, Álvaro Colom, haciéndole expresar "su profundo pesar". Los directores de los Institutos de Salud y los Centros de Control de Enfermedad (CDC, por sus siglas en inglés) calificaron este estudio como un

\footnotetext{
${ }^{3}$ Estudios experimentales en humanos de inoculación de sífilis, gonorrea y cancroide, y estudios experimentales en humanos de profilácticos contra la gonorrea, la sífilis y el chancroide a través de exhibición directa sexual e inoculación, nombres dados a dichos experimentos de acuerdo al Folder 1 Final Syphilis Report Part 1 records.
} 
hecho "lamentable y profundamente triste". El mismo Instituto de Medicina y la Comisión Presidencial de Bioética, ipso facto, programaron proporcionar informes de lo acontecido y de las protecciones recientes en vigor. Susan Reverby nos recuerda el ímpetu de los medios de comunicación sobre este caso, circularon en el mundo entero coberturas y editoriales de los principales periódicos. De hecho, ella menciona que "más de 150 mil historias fueron registradas" y que "su efecto sobre la protección de sujetos humanos en todo el mundo tendrá que ser evaluada en el futuro" 4 .

Los Archivos descubiertos por Reverby se hallaban en la Universidad de Pittsburg. Los documentos, actualmente, están disponibles físicamente en The National Archives, en Atlanta, y su versión digital está alojada en su página virtual (https://www.archives.gov/research/health/cdc-cutler-records). Esta versión virtual muestra ordenadamente las carpetas que contienen los informes de las prácticas experimentales de sífilis, gonorrea y chancroide, exámenes serológicos, el registro de pacientes, la correspondencia del Dr. Cutler, registros de aplicación de profilaxis, algunas revistas y una serie de fotografías ${ }^{5}$. Es importante mencionar que existe una copia de la versión física de los documentos en el Archivo General de Centroamérica, ubicado en la ciudad de Guatemala.

En lo que concierne a los estudios de sífilis, fueron empleados 726 sujetos de experimentación. De ellos, 696 (96\%) fueron expuestos a materiales infecciosos por coito o inoculación. De estos últimos, 427 (61\%) fueron considerados infectados y 369 (86\%) de ellos recibieron tratamiento con penicilina (CDC, 2010: 15-16). En cuanto a los estudios de gonorrea se utilizaron 772 sujetos, 234 (30\%) de estos fueron expuestos a materiales infecciosos y sólo 233 (99.5\%) recibieron tratamiento. En los estudios de gonorrea fueron empleados 142 sujetos, 138 (97\%) fueron expuestos a la infección y 129 (93\%) de ellos recibieron tratamiento (Comisión Presidencial

\footnotetext{
${ }^{4}$ Traducción libre de la versión original en inglés: "The media frenzy about this has circled the globe with coverage and editorials in major newspapers, and more than fifteen hundred stories have been filed. Its effect on the protection of human subjects around the world will have to be assessed in the future" (Riverby, 2011: 22).

${ }^{5}$ El Dr. Cutler al referirse a las fotografías expresa: "For the record, and to give credit where it is due, I should like to point out that most of these were taken by my wife, Eliese S. Cutler, who assisted as a volunteer in the project and used her own came[ra]s, as the project itself had none. She had training as a photographer" (Cutler, 1948: sin número de folio).
} 
para el Esclarecimiento de los Experimentos Practicados con Humanos en Guatemala, 2011: 28).

Según los registros sobre enfermos mentales, en el experimento se inoculó con sífilis a 638, de los que $423(66 \%)$ resultaron infectados. Para el $87 \%$ de estos (369) hubo un tratamiento adecuado, mientras que para el 3\% (10) solo hubo tratamiento parcial y, para el 10\% (44), no hubo tratamiento. De los 44 individuos sin tratamiento, dos abandonaron el asilo antes de ser tratados y uno murió antes del tratamiento (CDC, 2010: 15).

Se registraron 71 muertos durante los meses de observación prospectiva, estos 71 de 416 (17\%) correspondían a aquellos sujetos provenientes del hospital mental, ninguno de los 116 de la prisión. Los reportes no aseguraron relación de la muerte con los procedimientos del estudio. Sin embargo, pacientes que sufrían otras enfermedades no fueron excluidos del estudio; por lo menos, un paciente sufría de una afección 'pre-terminal' documentada al momento de la inoculación y falleció a los pocos días, así como otra persona con ataques, quien murió de epilepsia durante el último día de tratamiento de penicilina, aunque no queda claro si fue el tratamiento lo que precipitó los ataques incontrolables. En muchos casos la fecha de la muerte pudo haber sido registrada varios meses más tarde, y en muchos sujetos, no se anotó la causa de la muerte.

En su mayoría, se trabajó con los pacientes del hospital de salud mental con la mayor posibilidad de no ser dados de alta antes de las observaciones post-inoculación. De acuerdo con el Reporte Sumario de Sífilis, por la alta tasa de mortalidad en los internados en el asilo, sobre todo, por tuberculosis, se intentó hacer autopsias en todos los sujetos del experimento fallecidos, para estudios espiroquetales. Aunque se hicieron intentos de autopsia, sólo con 12 pacientes se anotó haberlo hecho (CDC, 2010: 20) y los resultados de las autopsias rutinariamente no fueron documentados.

¿En qué contexto es posible situar esta práctica de experimentación? Plantearse esta pregunta es importante para intentar elaborar una analítica del poder constitutivo y constituyente de la práctica misma. La base de esta "mecánica de poder" resulta ubicable en el marco del programa activo de investigación sobre el uso de la quimioprofilaxis para la prevención de enfermedades de transmisión sexual, el cual se desarrollaba en la década de los cuarenta en los Estados Unidos. Es decir, en ese entonces se perfilaban 
estudios para determinar el uso de preparados que podrían ser más eficaces y aceptables que el agente químico, de uso generalizado por los servicios militares de los Estados Unidos en la Segunda Guerra Mundial. Había interés en estos preparados tópicos alternativos, así como en el uso de la penicilina. Los estudios realizados en conejos, y aquellos estudios piloto entre los marinos de la Armada de Estados Unidos, daban indicios de resultados prometedores

En tal contexto, se consideró necesario elaborar estudios cuidadosamente controlados con grupos relativamente pequeños de individuos expuestos a un alto riesgo de infección, antes de proponer el uso generalizado de la nueva profilaxis -presumiblemente más eficaz y aceptable-, sobre todo, para los sujetos de las Fuerzas Armadas. Por lo tanto, la razón principal de los estudios en Guatemala consistía en desarrollar modelos de transmisión humana para determinar el impacto de los diversos regímenes quimio profilácticos, así como para redefinir una comprensión de la inmunología sifilítica en la era de la penicilina -especialmente, para una comprensión de la inmunidad a la reinfección de sífilis latente o recién curada (CDC, 2010: 10) ${ }^{6}$.

Habiendo expuesto, grosso modo, los hechos que constituyeron esta experimentación, este estudio toma distancia del resultado de investigación de los que le anteceden, mismos que están alojados en internet bajo títulos como: Public Health Sevice Sexually Transmitted Disease Inoculation Study of 19461948, Based on Review of Archived Papers of John Cutler, MD (CDC Report on Finding from the U. S., 2010); Éticamente imposible. Investigación sobre las STD en Guatemala desde 1946 hasta 1948 (Presidential Commission for the Study of Bioethical Issues, 2011); Investigación archivística sobre experimentos practicados en seres humanos en Guatemala, 1947-1948 (Archivo General de Centro América, Archivos de la Paz y el Archivo Histórico de la Policía Nacional, 2011); Experimentos con seres humanos, el caso Guatemala 1946-48 (Comisión Presidencial para el Esclarecimiento de los experimentos practicados en humanos en Guatemala, 2011); el informe Consentir el daño. Experimentos médicos de Estados Unidos en Guatemala (Comisión Presidencial para el Esclarecimiento de los Experimentos

\footnotetext{
${ }^{6}$ Confrontar con el Report on Finding from the U. S. Public Health Service Sexually Transmitted Disease Inoculation Study of 1946-1948, Based on Review of Archived Papers of John Cutler, MD, at the University of Pittsburgh
} 
Practicados con Humanos en Guatemala, 2011); y, por supuesto, el estudio ya mencionado que elaboró Susan Reverby (2011).

Sobre la base de los estudios mencionados, mi interés ha consistido en diagramar una hipótesis que se aproxime a lo subyacente de esta experimentación, cuya dimensión invoca, congrega y compete a humanos y no humanos en un régimen que podría denominarse tecnobiopoder, siguiendo a Haraway (2004b: 29). En este marco, describo esta práctica como un dispositivo de experimentación y, como tal, estará constituido por un conjunto de técnicas utilizables para la adquisición y la producción de cuerpos. Es interesante aproximarse a estas técnicas de gobierno de los cuerpos, sobre todo porque este dispositivo de experimentación tuvo lugar más "acá", al sur de Alabama, Estados Unidos, como ya lo señalaba Reverby ${ }^{7}$.

La reflexión que continúa se distancia pues, en primer lugar, de construir y habilitar una narrativa desde la victimización; y, en segundo lugar, de realizar una invocación normativa a los derechos humanos. Estoy consciente que, de realizar lo contrario a este distanciamiento, estaría reactivando, de mi parte, nociones humanistas antropocéntricas y refugiándome en los discursos paternalistas. Mi objetivo, al final, deserta de la fabricación de un listado de deberes morales para quedar bien con las figuras que podrían oxidar un aún por llamar "humanismo colonial".

\section{Gest(ac)ión de los cuerpos inviables para la humanidad}

Previo a desplegar algunas ideas sobre la particular forma que cobra el dispositivo de experimentación en los laberintos del incipiente fármacopoder en Guatemala, querría apuntar un par de anotaciones sobre el concepto mismo. El fármacopoder es un régimen, una forma de gobierno que motoriza sus vectores de poder a través del devenir fármaco del cuerpo y de la subjetividad. Siguiendo a Preciado podemos mencionar que:

\footnotetext{
${ }^{7}$ Traducción libre de la versión en inglés: "For Public Health Service researchers did, in fact, deliberately infect poor and vulnerable men and women with syphilis in order to study the disease. The mistake of the myth is to set that story in Alabama, when it took place further south, in Guatemala" (2011).
} 
El nuevo sujeto sexual fármacopornográfico emerge de una arquitectura científico-técnica que conecta elementos tan heterogéneos como barcos coloniales, testículos de ballena, soldados impotentes, recintos penitenciarios, embarazos de esclavas, textos bioquímicos y dinero. Las industrias farmacéuticas de principios del siglo $\mathrm{XX}$, que trabajan con una teoría experimental según la cual las hormonas se producen y se almacenan en las gónadas, se encuentran frente a la dificultad de procurarse grandes cantidades de ovarios y testículos (tanto animales como humanos). Los laboratorios farmacéuticos se instalan junto a los mataderos y "firman contratos con ellos para poder asegurarse una provisión regular de glándulas animales". En un intento de acabar con la penuria de extractos gonádicos, Alan Parkes, un fisiólogo inglés, admite haberse procurado "ovarios de una ballena azul" con la ayuda del Museo Británico (2008: 122).

En este sentido, el tráfico de fluidos y los microorganismos son elementales en los circuitos del fármacopoder. Por otra parte, considero útil alguna acotación sobre la necropolítica; es decir, el gobierno de los cuerpos y las subjetividades a través de una política que gestiona con la muerte. ¿Cómo se gobierna con la muerte? ¿Qué sucede cuando el poder de dar la muerte se convierte en un lento otorgamiento de la misma? ¿Qué ocurre cuando el dar la muerte lenta carece de espectadores? En todo caso, ¿y, si el dar la muerte ya no se asocia con el espectáculo y la instrucción, una especie de sintagma pedagogo-teatral propio del poder soberano, si le seguimos a Foucault en su lectura al poder soberano?

La cuestión de la relación entre fármaco y poder, al igual que la reflexión concerniente al gobierno de la vida y gestión de la muerte, vienen siendo trabajadas desde las ciencias sociales con una intensidad creciente en la región. Bianchi (2018) ofrece un panorama reciente y crítico sobre producciones teóricas e investigaciones empíricas, tanto a nivel global como en América Latina, provenientes del campo de las ciencias sociales y humanidades. Asimismo, con este artículo se recuperan estudios e investigaciones que ponderan al fármaco como fenómeno problemático e involucran diferentes actores, fuerzas, discursos, dispositivos, saberes, prácticas y tecnologías. Es más, el gobierno de cuerpos y subjetividades a través de políticas de gestión 
de la muerte viene siendo largamente trabajado por Susana Murillo. En diferentes trabajos aborda la problemática en términos de administración de la vida a través de la muerte (2015a), la muerte y el terror como técnica-táctica de gobierno (2018), y la exhortación a gestionar la vida y denegar la muerte (2008). En sus trabajos analiza diferentes ribetes de una matriz de gobierno de las conductas asentada en la amenaza de muerte y cómo se viene desplegando en la región (2015b).

En este dispositivo de experimentación pareciera no haber una puesta en escena de una espectacularización de la muerte, como sí se registraba en el antiguo poder soberano. Por otro lado, ¿cuál es la asociación de este otorgar la muerte lenta y oportuna con la deshumanización de los también humanos? ¿Cómo comprender la gestión de los cuerpos que "aún importan”? ¿Cuáles son las posibilidades, implicaciones, invocaciones y condiciones de un "aún importan"? ¿No se estaría diagramando el "aún” en términos de expiación y salvación apocalíptica? ¿Y para quiénes importan dichos cuerpos, y a qué precio? En el caso de los cuerpos en experimentación, para mí, el adverbio "aún" permite posibilidad, apertura, esperanza, expectativa...; pero también es un "aún", en tanto operable en concordancia con ciertos intereses, es decir, unos intereses proyectivos para no-dejar-ir sin ningún provecho o dejar-loahi mientras sea útil. Sirva entonces, este analisis micro-necropolítico para intentar problematizar las preguntas apenas esbozadas.

Los experimentos con estos humanos -o, mejor dicho, "inviables" como humanos- dan cuenta de la gestión de ciertos cuerpos, mismos que han sido implicados en los dramas de la salud y de la degeneración, cuerpos que no han tenido méritos en los "grandes" relatos de la familia, del parentesco y de la nación. Al confiscarlos fuera de lo que importa, permite leer esta gestión en los marcos del aparato de adquisición y el de producción corporal como cuerpos inviables, los cuales pueden ser legibles políticamente como encarnaciones de figuras paradójicas, en tanto que sus experiencias de vida/muerte podrían ser consideradas topos para una historia de la gest(ac)ión de los cuerpos anómalos en un cronotopo del tecnobiopoder. Por otra parte, su gestión puede permitir irrumpir la universalizada historia de la salvación, teniendo en cuenta las 
misiones civilizadoras del colonialismo cristiano que performa un llamado "Nuevo Mundo".

En este sentido, este relato que delineo puede mostrar la parentela de estas siervas y siervos sufrientes (Haraway, 2004a: 47-61) con los conejos y con la oncoratona (Haraway, 2004b: 99-106) dentro de la arquitectura de manufacturación del laboratorio. Estas figuras, habitantes de un mundo por venir, encarnan las figuras del realismo cristiano, en tanto que cohabitan la historia de la salvación, pero también la de la comedia. Estos cuerpos constituyen, en lenguaje de Haraway, en tanto "textos y artefactos dentro de la tecnociencia", una figuración que "es apocalíptica y cómica a la vez" (2004b: 25). Estas escenificaciones rememoran la historia de las operaciones de deshumanización del colonialismo ibérico, mismas que dieron materialidad a las violencias originarias contra los cuerpos extraños; esos indixs que amenazaron las preocupaciones coloniales primigenias sobre el cuidado de la raza, de la "buena familia" y, posteriormente, de la nación. Tengo la certeza de que el recuento de esas operaciones deshumanizantes de lxs otrxs degeneradxs finalmente refuta como objetivo el invocar una integración o asimilación a la gran "familia humana" a la nación o a las ciudades higienizadas. Esas heterotopías que contienen escáneres de reconocimiento de especie, de género, de sexo, y de raza. Es decir, a aquella institución que con Gabriel Giorgi podríamos llamar la gran familia de inclusión social y biopolítica (2013: 11).

Una interrogante que podría ser más bien el leitmotiv de esta narrativa que estoy fabulando sería: ¿Qué es lo que cuenta como humano en esta escena biomédica etnoespecíficamente localizada? El cuerpo de la prostituta, el del

\footnotetext{
${ }^{8}$ Aquí sigo una cita textual de Haraway, quien, al hacer una aproximación al humanismo, menciona que los eventos del proceso de Jesús relatados en el evangelio de Juan probablemente no sean históricos, sino teatrales. Haraway sostiene que "desde el comienzo se escenifica la historia de la salvación, la cual se vuelve el modelo de la historia del mundo en las herejías seculares de los siglos del colonialismo europeo, con sus misiones civilizadoras y discursos genocidas sobre la unidad de la humanidad". Traducción libre de la versión inglesa: "John staged the trial before Pilate in terms of the suffering-servant passages from Isaiah. The events of the trial of Jesus in this nonsynoptic gospel probably are not historical, but theatrical in the strict sense: from the start, they stage salvation history, with then became the model for world history in the secular heresies of the centuries of European colonialism with its civilizing missions and genocidal discourses on common humanity" (2004b: 50).
} 
enfermo mental, el del preso, el del soldado y el del niño fueron vehiculizados como cuerpos "anómalos", convocados en las arquitecturas de la salud y de la degeneración para las operaciones de la reparación y el aumento de las condiciones materiales de la existencia de los, así llamados, "cuerpos soberanos". Es decir, aquellos cuerpos que se auto delegan el derecho de vivir y morir dignamente. Es así que los cuerpos "anómalos" son conducidos a la inteligibilidad en los discursos de la medicina y de la salud de quienes se auto convocan en las fronteras de las vidas vivibles y de las utopías de las vidas eternas.

Sigo a Mbembe al cuestionar que "si consideramos la política como una forma de guerra, debemos preguntarnos qué lugar deja a la vida, a la muerte y al cuerpo humano" (2006:20). También sigo su argumentación cuando rescata lo que Foucault entendía por el biopoder, es decir, aquel dominio de la vida sobre el que el poder ha establecido su control. Por consiguiente, al situarme en este experimento, planteo la pregunta: ¿en qué condiciones concretas se ejerce ese poder de matar, de dejar vivir o de exponer a la muerte? Esos cuerpos de experimentación habían sido despojados de su humanidad y, con ello, ensamblados directamente a un estatuto ontológico, asimétricamente desigual, que los dispone para la expoliación y el despojo. Me parece genuino subrayar aquí, y a partir de aquí, lo que Mbembe afirma cuando reescribe lo que Frantz Fanon afirmaba al referirse a la "ciudad del colonizado" como un topo en el que "se nace en cualquier parte, de cualquier manera" y en el que "se muere en cualquier parte, de cualquier cosa", in fine, "una ciudad revolcada en el fango" (2006: 45). Es decir, que las técnicas de despojo utilizadas para gestionar el cuerpo de los desterrados de la humanidad se imbrican con las elaboraciones discursivas e imaginarias de unos cronotopos del tipo todo-puede-pasar-ahi.

\section{Tecnología de adquisición y producción corporal}

En este apartado, querría traer a colación la técnica de poder griego que consistía en lo que Chamayou (2012) denominó "atrapar hombres (sic)"; es decir, aquella que se refiere a la tecnología de la captura ${ }^{9}$. Para este caso de

\footnotetext{
${ }^{9}$ Una tecnología que también tiene efectos en la cacería de mujeres jóvenes para conducirlas a los burdeles a finales del siglo XIX en Guatemala. Esta cuestión está descrita en este ensayo: Prostitución como 'mal necesario' a finales del XIX en la Ciudad de Guatemala: sexopolitica, pornotopía y supremacía masculina. Tal ensayo forma parte de la compilación de AVANCSO:
} 
análisis, probablemente, dista de ser una tecnología propiamente cinegética. En la antigua Grecia, el miedo del griego a ser cazado era muy importante y, podría decir, que como una "tecnología del yo" (Foucault, 1990: 45-47), por citar al filósofo francés. Como afirma Chamayou, se temía mucho a un personaje denominado andrapodistes. Este era, literalmente, un cazador de hombres que se apoderaba de los ciudadanos al capturarlos, para luego venderlos como esclavos. En ese sentido, el miedo griego consistía en temer al estatuto ontológico de ser esclavo. En el caso de la práctica del experimento es preciso señalar que el Dr. Cutler y sus colaboradores fueron secuestradores de hombres y mujeres, pero también diseñadores del nuevo estatuto político de los sujetos. Hacían uso del estatuto des-ciudadanizado de los sujetos, desincorporados de la ciudadanía, es decir, de su incapacidad de ser buenos ciudadanos para transformarlos en sujetos renovados para las prácticas quimioprofilácticas. Por otra parte, Chamayou nos recuerda que "el griego se apodera de sus esclavos como lo hace de una presa en la caza o de las frutas en la recolección: es decir, sin tener que organizar su producción” (2012: 13). Esto implica una tecnología de despojo de todo lo dispuesto, lo disponible sin tener que hacer muchos esfuerzos, pues ya existe un campo abierto para tal operación y un estatuto de disponibilidad de los cuerpos. Es decir, el poder de dar la muerte se convierte en una operación alargada, procesual, liminal de quienes han sido capturados y des-ciudadanizados, cuya proximidad a la muerte no tiene nada que instruir a los espectadores. Al parecer, la captura de estos cuerpos en el laboratorio de Cutler responde a este proceso dual.

La primera captura obedece a la "anormalidad" de estos cuerpos, en tanto naturalizados como criminales, enfermos, locos, no adultos y perversos sexualmente. La segunda captura responde a una utilidad por el "bien”, es decir para conseguir la buena salud, aunque no la de ellxs, por lo que el ejercicio de esta violencia no llega a ser exactamente "una violencia por tu propio bien”. Más bien, es una violencia por el bien de quienes son catalogados como receptores de la "buena salud". Es aquí donde una técnica propiamente de discapacitación del viviente humano transita hacia una sobredosificación de su potencia para los tentáculos del fármacopoder. En tanto que, puesta en

Producción corporal. Interfiriendo engranajes de violencia en Guatemala (Chivalán, 2019: 1-36). 
escena de un poder cinegético, en esta práctica todos los cuerpos pueden morir, pues son reducidos al ámbito de lo recursivo. Y, como afirma Chamayou, en caso de pérdidas siempre habrá otros recursos, otros cuerpos que capturar. En tanto que los cuerpos experimentales responden a una recién mencionada doble captura, es posible esbozar que la primera captura responde a la técnica de adquisición corporal recursiva y, la segunda, en tanto re-capacitación del cuerpo, responde a una técnica de producción corporal.

A continuación, deseo escenificar el drama que implica un aparato de producción corporal de los cuerpos capturados en los relatos de la historia de la salvación para los cuerpos soberanos, es decir, los "cuerpos que sí importan”. Como mencioné anteriormente, el Dr. Cutler, junto a su séquito de colaboradores, se traslada al hábitat de la especie por cazar. Esto debido, por una parte, a las condiciones de un cronotopo del todo-es-posible, como es el caso de Guatemala; y, por otra, al imaginario aún bastante extendido de la sociedad guatemalteca de que todo lo proveniente del norte global es de mejor calidad y de mayor autoridad.

En estas condiciones es posible "aprovechar" los cuerpos considerados "naturalmente" anómalos y despojarlos dentro de la arquitectura del fármacopoder. En este caso, no se trata de percibirlos como enemigos y, por consiguiente, eliminables, como en los relatos sobre el genocidio reciente en Guatemala. Sus existencias -el estatuto de cuerpo vivo que encarnan- no representan un "atentado", una "amenaza mortal" o un "peligro absoluto". La gestión de estos cuerpos tiene más que ver con la mutación de su estatuto político recursivo. Estos cuerpos capturados para el laboratorio son biofísicamente metamorfoseados, son potenciados para el hábitat del conejillo de indias, cuerpos farmacológicamente controlados, ensamblajes humanobacteriológicos para el desarrollo de las ciencias de la salud y de la "vida sana y pura”.

Estos cuerpos obturados y diseccionado por la práctica dela experimentación vivencian la triple pérdida de la que habla $\mathrm{Mbembe}^{10}$ : han perdido su hogar,

\footnotetext{
${ }^{10}$ Hay que tener presente que es riesgoso extrapolar el análisis de Mbembe, sobre todo, porque su caso de análisis se circunscribe a la esclavitud en los registros de la necropolítica, pero sí merece la pena ponderar la importancia de esas pérdidas, las cuales también son propias de los cuerpos dentro del fármacopoder.
} 
han perdido los derechos sobre su cuerpo y han perdido su estatuto político -mejor dicho, han transmutado su estatuto político-. Esta triple pérdida, de dominación absoluta y muerte social, según Mbembe, equivale a "una expulsión fuera de la humanidad" (Mbembe, 2006: 32). En ellos se registra una expulsión a la crueldad y al horror.

Esta analítica de producción corporal concierne a la mutación de cuerpos cuasi-humanos -anómalos, inviables, incompletos, inadecuados... como humanos- a cuerpos farmacológicamente bacterianos, recodificados como humanos. Esta práctica de experimentación con humanos, considerada como dispositivo de deshumanización por Chamayou, puede ser leída también como un aparato de producción corporal; es decir, los cuerpos que somatizan la sífilis, la gonorrea, el chancroide y los diagnósticos serológicos podrían ser considerados "cuerpos viles", haciendo alusión a otro trabajo de Chamayou (2008). Sin embargo, también me parece oportuno pensar que la gestión de dichos cuerpos reactualiza el orden colonial. Propongo que en esta experimentación se yuxtaponen mecanismos propios de la empresa colonial y cristiana, confabulando con un aparato de producción corporal de doble función. En primer lugar, opera como una técnica de producción corporal discapacitante de lo humano. En segundo lugar, opera la función de sobre-cualificación corporal al transformar estos sujetos en humanos redentores. En el caso particular de este estudio, se sedimenta este proceso: de una "disfuncionalidad" humana, se transita a un inmediato capacitismo farmacológicamente aprovechable.

Estos cuerpos de experimentación han sido considerados no lo suficientemente humanos, por lo que, desde un humanismo bíblico, occidental, varonil, racista, colonialista y cristiano es posible hacer de ellos, oximorónicamente, redentores de aquellos que "sí importan". El dispositivo de experimentación los transforma en rescatadores de aquellos para quienes las diferencias sí importan, únicamente en tanto que esos cuerpos sean aprovechables y serviles para los proyectos de salvación de ellxs como buenxs ciudadanxs, aquellxs que pertenecen a las mejores familias y a las prósperas naciones. Encuentro aquí, por consiguiente, un proceso de "reciclaje", puesto que aquellos cuerpos que han sido considerados inviables dentro del 
humanismo ya mencionado son reparados en una determinada fábrica de producción corporal: ¡ Resarcimiento de los cuerpos inviables para el bienestar de los viables! En este caso se cimenta una técnica de producción en la que ciertos cuerpos son "naturalmente" considerados extraíbles y despojables para la acumulación de la vida en el marco de la inteligibilidad biopolítica de lo humano.

Este aparato de producción corporal podría formar parte de una "historia tecnopolítica" (Chamayou, 2008: 383) de la subjetividad colonial, pues trata de converger las técnicas de adquisición de cuerpos biodespojables para la producción farmacológica. Es decir, la producción de cuerpos "venéreos" para la experimentación médica y farmacológica. Una práctica que registra, ni más ni menos, una violencia excesivamente micropolítica ejercida sobre "estos" sujetos, que podrían ser los que Judith Butler describe al mencionar que existen sujetos que han sido "constituidos por la exclusión, es decir, por la creación de un dominio de sujetos no autorizados, de presujetos, figuras de abyección" (Chamayou, 2008: 19) ${ }^{11}$.

Mi reflexión sigue también a Paul $B$. Preciado, quién a través de un programa de investigación y docencia ha propuesto aproximarse al cuerpo como un "archivo político y cultural". Él toma distancia de las aproximaciones al cuerpo como "corpus, templo, sede del poder soberano, envoltorio, domicilio o propiedad". Para Preciado, filosóficamente, "el cuerpo moderno (sano o enfermo, normal o patológico) no tiene estatuto ontológico, sino biopolítico y performativo" (2012). El cuerpo no existe fuera de:

[...] un conjunto de prácticas discursivas, epistemológicas, científicas, farmacológicas, económicas, mediáticas y visuales, a través de las políticas de inmigración y de gestión del riesgo, de ensayos clínicos, de técnicas farmacológicas, de prácticas de diagnóstico, de narrativas mediáticas, de entramados discursivos, de representaciones visuales y de discursos de prevención, control y vigilancia. Dicho de otro modo, los discursos médicos,

\footnotetext{
${ }^{11}$ Palabras de Judith Butler citadas por Chamayou. Quiero mencionar que una tecnopolítica de este tipo ha de reclamar, para el aggiornamento del orden colonial en Guatemala, una filosofía política del modelo extractivista o de la acumulación por biodesposesión de humanos y no humanos, irrumpiendo nostalgias y proyectos antropocéntricos.
} 
políticos y audiovisuales que representan el cuerpo producen la normalidad o la patología que pretenden describir (2012).

Con esta cita, y enfocando en mi caso de estudio, propongo una ruptura con este aparato de producción corporal farmacológicamente controlado, puesto que para la elaboración de la norma de lo humano y del cuerpo nacional, se reactualiza una visión colonial de lo humano, visión no ajena en la historia pasada y presente de Guatemala. A mi juicio, la imbricación de la técnica de adquisición y la técnica de producción corporal en la experimentación, hizo entrar en juego la "norma" de lo humano y el estatuto político del viviente. Postulo que esta imbricación pertenece al ámbito del fármacopoder y a la necropolítica, como lo denominaríamos con Preciado leyendo a Agamben. Las "cobayas humanas" en este paisaje han sido replegadas a la "vida desnuda", al ser objetivados desde una explotación farmacológica (Preciado, 2008: 43) ${ }^{12}$. En este sentido, preciso reescribir lo que Giorgio Agamben menciona, que si en todo Estado moderno "hay una línea que marca el punto en el que la decisión sobre la vida se hace decisión sobre la muerte y en que la biopolítica puede, así, transformarse en tanatopolítica, esta línea ya no se presenta hoy como una frontera fija que divide dos zonas claramente separadas" (2010: 15556). Los cuerpos que se auto perciben como soberanos son los beneficiarios de la desposesión de lo "aún humano" de aquellos cuerpos de experimentación. La gestión del viviente cuasi humano, en esta lógica y esta praxis, se convierte en condición material y recursiva de la existencia biopolítica de los cuerpos "soberanos". Por otra parte, también es posible inscribir esta tecnología como colonial, puesto que constituye cuerpos y subjetividades discapacitados para gestionar técnica y políticamente su muerte a través de un proceso tecnoextractivo, nada sorpresivo en el paisaje colonial. Este aparato ensambla cuerpos diferenciales donde las diferencias parecen no importar.

Pienso, con Teresa de Lauretis, que la producción corporal anómala, monstruosa, incapacitante $y$, paradójicamente, potencial para la experimentación como producto y proceso de un conjunto de aparatos tecnosociales, biomédicos y biofármacos significa haber superado ya a Foucault,

\footnotetext{
${ }^{12}$ Aquí sigo una cita textual de Preciado: “[... lo que caracterizaría a aquellos que según Agamben se ven reducidos a 'vida desnuda' tanto en las sociedades democráticas como en los regímenes fascistas es precisamente poder ser objeto de una explotación farmacopornográfica” (2008: 43).
} 
pues su concepción crítica de la tecnología del sexo olvida la solicitación diversificada a la que ésta somete a los sujetos/cuerpos masculinos y femeninos (2000: 35). Recalco aquí que una tecnología colonial es una también de producción corporal diferencial. La producción bio-corporal diferencial -en términos raciales- a partir de la pregunta que indaga por el alma deviene en una tecnología de diferencia racial y cualidad humana. La experimentación, como mecanismo de extracción biomédica, opera acorde a un modo del nodejar-morir-del-todo para la mejora de las condiciones materiales de vida y de existencia de los corresponsablemente humanos. De aquí, me parece ineludible efectuar un paralelismo, más no una analogía, para lo cual sigo la citada aclaración de Hazel Carby por Haraway, concerniente a las operaciones en el Nuevo Mundo, al mencionar que, en los Estados Unidos, particularmente, "las mujeres negras no fueron constituidas como 'mujeres', del mismo modo en que sí lo fueron las mujeres blancas” (2004a: 55-56) ${ }^{13}$. Para el caso de los cuerpos colonizados, algunos han sido constituidos como humanos no plenos -en el caso de no haber sido producidos para la muerte física y simbólica en los genocidios tempranos en el así bautizado Nuevo Mundo ${ }^{14}$ - y como tal, sí gestionables desde una política del biodespojo y de la extracción. Por otra parte, me parece útil referirse a lo que Foucault aducía al decir que el sueño de los poderes modernos es el genocidio (Foucault, 1995: 166) ${ }^{15}$. Aun así, este no

\footnotetext{
${ }^{13}$ Traducción libre de la versión en inglés: "Hazel Carby clarified in the New World, and specifically in the United States, black women were not constituted as 'woman.' as white women were".

${ }^{14}$ Más bien, fines del mundo para muchos humanos y no humanos.

${ }^{15}$ Hay que tener en cuenta que el genocidio para el caso de Guatemala -perteneciente a su historia reciente- fue la forma máxima en que se sedimentó el racismo de Estado. Por otra parte, la época conocida como los diez años de primavera guatemalteca corresponden con los años de 1944-1954, en los que, como Reverby hace notar en su trabajo, tenía importancia la United Fruit Company en una República bananera como la guatemalteca: "The United Fruit Company owned and controlled much of Guatemala, the quintaessential 'banana republic,' in the first half of the twentieth century. When the PHS looked to Guatemala for its research in the inmediate post-World War II years, it came into the country during the period known for its relative freedoms. Between 1944 and the U.S.-led CIA coup of the elected government in 1954, efforts were made at labor protection laws, land reform, and democratic elections. The PHS was a part of the endeavor to use Guatemala for scientific research as it presumed to transfer laboratory materials, skills, and knowledge to a Guatemalan Public health elite (Riverby: 2011: 11).
} 
tiene que ver con el retorno al "viejo derecho a matar"; más bien, se debe a que "el poder reside y ejerce en el nivel de la vida, de la especie, de la raza y de los fenómenos masivos de población” (1995: 166). Pienso en los cuerpos infectoinoculados, en los que parece haber una confabulación entre las industrias de la muerte, sean éstas farmacológicas, bélicas u otras. Es importante hacer hincapié en este tipo de correlaciones, pues, esto también, me permite pensar la imbricación permanente entre las variadas técnicas de poder que gestionan el cuerpo y la subjetividad en un particular topo situado llamado Guatemala.

La experimentación se realiza también en un marco de simbiosis soberano, si seguimos a Agamben (2010). En palabras de este filósofo, se produce un marco en el que el jurista, el médico y el especialista disponen de cuerpos degenerados -vivientes-muertos o moribundos-vivos- para las operaciones de la tecnología del despojo. En este sentido, propongo especular sobre el "cuerpo reciclable", sin intereses ontológicos, sustancialistas u organicistas. Me resulta sugestivo pensarlo en términos de ficción somática y técnica, a partir de la cual elaborar pedagogías críticas para otros cuerpos "indecorosos". Estos cuerpos, concebidos como prótesis, extensión o cura, participan, a su pesar y con altos costos como la muerte, en la construcción de la norma de lo humano viviente. En dicho ensamblaje se diseña una especie de "humanismo protésico" para su exposición y/o disposición a las prácticas in vivo de fármacos y enfermedades. Al conseguir testar los fármacos o comprobar las curas, esos fármacos o las técnicas de la cura se extienden al conjunto de los cuerpos dignos de vida. Las vidas indignas de ser vividas son dignificadas únicamente en tanto que otorgan garantía de vida a aquellos que cuentan con una vida digna de vivir. Parece, entonces, que es posible visibilizar que el poder de dar la muerte se confunde dentro de las coordenadas biopolíticas en tanto gestión de la vida indigna para la mejora de la salud de una población selecta.

Esta analítica parece mostrar que se entremezclan los cuerpos deshumanizados con quienes sí merecen vivir dignamente, en tanto que los humanizados devienen generadores de longevidad, salubridad y extensión de las vidas. Estos cuerpos humanizados y reciclados son plusvalorizados para las empresas del tecnobiopoder, sean éstas de la guerra, de las industrias biofarmacológicas o de las bioextracciones. Sigo a Agamben cuando sostiene que "la 'vida digna de ser vivida' no es un concepto político" -aunque pareciera ser lo evidente- al referirse a los legítimos deseos y expectativas del 
individuo: sino más bien, "es un concepto político en el que lo que se pone en cuestión es la metamorfosis extrema de la vida eliminable e insacrificable del homo sacer" (2010: 179). La biopolítica, desde esta lectura y al referirse a los sujetos en la experimentación dentro del aparato de producción corporal, se transforma en necropolítica porque la "vida nuda" se sitúa en la encrucijada de la decisión soberana sobre dicha vida inviable, suprimible impunemente, y aquella asunción que tiene que ver con el cuidado del cuerpo biológico y social de la población privilegiada en los paisajes de un humanismo aún presente.

Me parece sugestivo seguir profundizando esta noción para poder aproximarse a la elaboración de un relato que permita, conjuntamente con los cuerpos de experimentación, otras ficciones con las cuales gobernarnos; es decir, otras contra-narrativas y prácticas de insubordinación somática y política, de insubordinación simbólica y material para imaginar epistemes y alteridades significativas (Haraway, 2016: 19-20) aún posibles, resistiendo a cualquier aparato de producción corporal normativo y dominante.

\section{Ficción especulativa a modo de conclusión: Indianimalia}

"La deshumanización teórica y práctica del enemigo no es más que uno de los usos políticos posibles del concepto de humanidad, pero no el único". Grégoire Chamayou (2008)

Chamayou ha planteado que una de las técnicas dominantes en la historia de la experimentación humana ha sido la degradación de quienes han sido considerados sujetos de esa práctica. El mismo autor argumenta que las técnicas de degradación surgen como un envés de las técnicas de valorización. Es decir, que todo aquello que produce lo humano converge en un conjunto de tecnologías de humanización. En cambio, las técnicas de degradación siguen un proceso inverso, al converger en unas tecnologías de deshumanización ${ }^{16}$. Es fundamental para Chamayou el abordaje de las técnicas de degradación, en tanto que su tesis se vincula directamente con el análisis de la experimentación. Él arguye que "una de las formas dominantes de tecnologías de la adquisición en

16 Traducción libre de la versión en francés: "Les techniques d'avilissement apparaissent au contraire comme étant l'envers de techniques de valorisation, 'l'humain' étant le produit d'un ensemble de technologies d'humanisation tout comme l'est, dans un processus inverse, sa déshumanisation" (Chamayou, 2008: 18). 
la historia de la experimentación humana ha sido la degradación de los sujetos de la experiencia, sea que esta degradación haya sido directamente iniciada por el experimentador o que haya preexistido en el curso experimental como una externalidad disponible de la cual podría sacar partido" (ídem: 17) ${ }^{17}$.

Por consiguiente, Chamayou considera que existen técnicas que humanizan y técnicas que deshumanizan. Si se conciben estas técnicas como opuestas, o técnicas de inverso y reverso, probablemente se estaría adjudicando estatutos ontológicos a los procesos de producción de dichas técnicas. En otras palabras, las técnicas que humanizan siempre estarían humanizando, pero, entonces, ¿cómo se concibe lo humano? ¿Cuál es la medida de una humanidad plena? En este contexto es importante recordar que la investigación de Chamayou se inscribe en una gama de trabajos que, según él, podrían ser considerados estudios críticos de las subjetividades degradadas.

Volviendo al planteamiento que indaga por el estatuto ontológico de quién cuenta como humano y quién no, Chamayou expresa que en un procedimiento retórico habrá que jugar el sentido del término 'humanité' (humanidad). En la medida en que no concierne ni al carácter ontológico común a todos los hombres (sic), ni como una totalidad, en tanto que conjunto de hombres (sic), dentro de una sucesión de generaciones. Lo que para él es "la humanidad" concierne únicamente a una cualidad contingente: "La humanidad no es tanto más la esencia de un ser sino la cualidad contingente que recibe un agente en función de sus intenciones" (ídem: 73) ${ }^{18}$. En este contexto, si el criminal actúa en beneficio del género humano, deja de ser inhumano. Sobre este postulado me interesa matizar que tal inhumanidad se refiere a la experiencia a la que han sido arrinconados los cuerpos de experimento, como agentes de crimen o agentes contaminantes de la pulcritud racial, y no por ser, ontológicamente, inhumano.

\footnotetext{
${ }^{17}$ Traducción libre de la cita original en francés: “[...] l'une des formes dominantes de technologies d'acquisition dans l'histoire de l'expérimentation humaine a été l'avilissement des sujets de l'expérience, que cet avilissement ait été directement initié par l'expérimentateur ou qu'il ait préexisté à la démarche expérimentale comme une externalité disponible dont il pouvait tirer parti”.

${ }^{18}$ Traducción libre de la versión francesa: “L'humanité n'est plus l'essence d'un être, mais la qualité contingente que reçoit un agent en fonction de ses intentions".
} 
En este caso, es importante para mí considerar que los sujetos de experimentación en los estudios realizados por el Dr. Cutler y compañía entran a formar parte de una categoría degradada de ser humano; sin embargo, planteo que estos degradados, en cierta manera, se convierten en sujetos biodespojables al ser reducidos a cuerpos-recursos -archivos en los que se inscriben política y farmacológicamente como una utilidad para el beneficio de la humanidad; es decir, de la humanidad de ciertos cuerpos que sí importan dentro de los límites de un Estado-nación y su periferia: cuerpos que importan para la (re)producción del cuerpo nacional sano de la población militar estadounidense, en este caso-.

Chamayou, al referirse a los cuerpos condenados, recuerda que, paradójicamente, se encuentran en un espacio de no-libertad, el espacio de la prisión. Al mencionar que, en los experimentos médicos realizados en los condenados, ellos ya no pueden decidir, pues están ya considerados cuerpos para la muerte. Sin embargo, en el caso de Guatemala, los presos y los locos (que también fueron involucrados en las experimentaciones) no solamente estaban en esa condición de no-libertad, sino que la disposición de sí mismos no formaba parte de sus decisiones. Ni el doctor Cutler de los Estados Unidos ni el doctor Funes de Guatemala solicitaron su autorización ni su permiso para intervenirlos. Fueron las instituciones aseguradoras de la salud pública las que realizaron intervenciones en esos cuerpos a partir de la autorización de los médicos y directores de esos centros, acreditados a su vez por la respectiva institución estatal de Guatemala.

Lo que Chamayou describe en su texto acerca del cuerpo de los condenados, se concretiza en Guatemala a mediados del siglo XX: el equilibrio utilitarista entre la vida de algunos hombres y el progreso de la humanidad entera como especie da lugar aquí a un verdadero negocio de deshumanización: la vida de un criminal, como la de un loco, no vale nada o vale únicamente dentro de los cálculos biopolíticos de la salud y de la cura. En esta argumentación, la deshumanización de los cuerpos viles aparece como el corolario retórico de su explotación. Es decir, que el criminal purifica su crimen hacia el bien público al sanar el cuerpo digno de vida. Sin embargo, en el caso de Guatemala, el criminal no expía su crimen, su cuerpo es utilizado porque ya era inviable per se como humano y, así, desincorporado del espacio público y adjudicado a un 
espacio privado de no-libertad. El loco, a su vez, no tiene frente a sí horizonte de cura alguna, es un insano, y por eso se une al criminal en la imposibilidad, no ya de expiación, sino de retorno de la locura. El loco encarna lo que Foucault denominó el residuo de los residuos (Foucault, 2005), y que distintos autores, que van desde el propio Foucault a Descombes, pasando por Rose y Huertas, hasta Canguilhem y Goffman, han posicionado en el centro de los estudios que articulan saber, poder $\mathrm{y}$ verdad desde las ciencias sociales y humanidades (Bianchi, 2019).

De los casos que cita Chamayou acerca del uso del cuerpo de los condenados para experimento, resulta interesante traer a colación un caso individual, referente a Keanu, un hawaiano condenado a muerte a quien en 1884 se le inoculó lepra. Este caso fue particularmente debatido debido a la preocupación por la degradación de la ciencia y no la de Keanu. No importaba el cuerpo de Keanu; al fin y al cabo, él no tenía otra opción que la muerte. La preocupación radicaba en la degeneración de la ciencia a partir de experimentar sobre un cuerpo otro.

Los principales debates tienen que ver con las técnicas de degradación de los sujetos de experimentación. Chamayou realiza una lectura política de las prácticas de experimentación en tales siglos. El mismo autor describe todos los debates médico-jurídicos, filosóficos y deontológicos en torno a tales experimentos. Dichos debates han discernido, por ejemplo, sobre la posible bestialización del humano a través de las técnicas de vacunación, provenientes de la viruela de vaca o viruela vacuna contra la viruela humana. Este debate tuvo lugar cuando Edward Jenner descubre la vacuna en 1796. Se debate sobre si tal práctica daría origen al embrutecimiento de la población, además de la animalización de los humanos.

Se plantea como problema el traspaso de fluidos de las vacas a los hombres. Sin embargo, se buscaba también ansiosamente la cura de la viruela, pues concierne al control de la población dentro de las coordenadas del cálculo y la aritmética política. La población es concebida desde una biopolítica de la estadística. También se discurre sobre la inoculación humana al intentar buscar procedimientos que fuesen de transmisión de hombre a hombre. Marcus Herz, amigo de Immanuel Kant, introduce la posibilidad de un procedimiento basándose en el principio de la homogeneidad y la identidad intra-específica. La materia humana en la que se piensa es la del cuerpo 
del extranjero. Situarse en estos análisis sobre la animalidad, la bestialidad, el embrutecimiento, la acuñación de un cuerpo extranjero a la humanidad de estas poblaciones conlleva nuevamente a preguntarse por la norma de lo humano. ¿Qué es y quién es este humano inmutable y sustancial, inmune a la contaminación animal y colonial? En este contexto, es preciso traer a colación otro caso paralelo, referente al momento cuando se discutía el sacrificio de vidas individuales para salvaguardar la salud promedio de la población.

Teniendo en cuenta el panorama anterior, Chamayou postula que en ese momento surge una tensión entre cálculo político y ley moral. Aquellos adversarios a la inoculación aseguran que "la vida salvada de miles de ciudadanos no justifica la muerte de uno solo [...] el mal de uno no se repara, no expira por el bien de los otros" (2008: 126) ${ }^{19}$. Asegurar la vida de miles de ciudadanos por la muerte de uno solo. Sobre tal argumento, me inquieta nuevamente la cuestión que inquiere sobre cuáles vidas deben ser salvadas y cuáles sacrificadas por el bienestar, la longevidad y la salud de los "buenos ciudadanos" estadounidenses de la Posguerra, en el caso de experimentación en Guatemala.

Otra escena, sobre la cual ausculta Chamayou, es la referente a la experimentación colonial. El filósofo indica que los proyectos que incumben a este tipo de experimentación tienen que ver con tres direcciones, las cuales son: una raciología experimental, una medicina racial y una teoría de la aclimatación (ídem: 349), -escena que también es revisada por la filósofa e historiadora Elsa Dorlin en Medicina colonial y medicina esclava (2009: 231-243)-. La pregunta que me inquieta en este apartado es la que indaga por cuáles son los cuerpos dignificados para la experimentación y cuál es la población que debe ser salvada.

Conducido por el estudio de Chamayou, me percato sobre una serie de planteamientos vinculados con las enfermedades propias de los colonizados. En el caso de las colonias francesas, los experimentos proyectados sobre los cuerpos colonizados exigían complejos debates. Los colonizadores se enfermaron durante la invasión y era necesario salvaguardar sus vidas para alcanzar los objetivos de sus empresas coloniales. En este contexto, la teoría de la aclimatación representaba una doble cuestión o una paradoja. No solamente había que temer que el colono se tropicalizara, también se

\footnotetext{
${ }^{19}$ Traducción propia de la versión francesa: "La vie sauvée à mille citoyens ne justifie pas le meurtre d'un seul. [...] le mal de l'un ne se répare, ne s'expie point par le bien des autres".
} 
consideraba necesaria su tropicalización para adaptarse e inmunizarse de las enfermedades consideradas propias de los colonizados. Al adaptarse al trópico, el colono estaría más preparado para seguir con sus empresas de colonización. Por otra parte, los experimentos por realizarse en los colonizados planteaban el complejo debate correspondiente a si la humanidad del colonizado era equiparable a la humanidad del colono. ¿Eran traducibles las conclusiones de los experimentos en los colonizados a la humanidad de los colonos? Aquí se tropieza de nuevo con la pregunta que escudrińa la medida de la humanidad.

El escrutinio por el alma de los indios, para el caso de América, ha generado una justificación de una serie de atropellos, despojos y desarraigos, porque la respuesta a esa inspección ha sido el dudoso otorgamiento de una humanidad desde ya diferenciada. Una humanidad diferenciada que ha de justificar - ¿ante quién?- los abusos y usos de unos cálculos económicos ensayísticos que empezaban a producirse. La vida y la reproducción misma de los indios parecieron quedarse desde ya confiscadas dentro de un aparato de producción corporal. La diferenciación que se generó en esos tiempos ha sido utilizada para la distribución espacial de los cuerpos. De ahí, la producción de un afuera/ adentro territorial que nos recuerda el cuidado/profilaxis de la pureza racial.

Considero útil esbozar una somera especulación sobre el concepto de la indianimalia, como concepto político. En este sentido, es posible discernir las contradicciones que constituyen lo humano en este dispositivo de experimentación y, como tal, darle una relevancia histórica. Se precisa recordar que, bajo el mismo paraguas del humanismo, se dirimió una lucha sobre la colonización del así llamado Nuevo Mundo. Chamayou (2012) recuerda que este debate se acogió bajo esta contradicción del uso conceptual del humanismo. Tanto los partidarios de la conquista violenta, como los defensores de los indios, apelaban al mismo concepto de humanismo, ya sea para justificarlo o para condenarlo.

Me resulta útil pensar en la arriesgada yuxtaposición de colonización y humanismo de acuerdo al caso analizado. Asumo que un anacronismo de este tipo puede resultar peligroso, pues podría dar lugar a un transhistoricismo de última hora. Pero, sí me parece válido intentar problematizar la conjunción entre humanismo y colonialismo, quizás porque permite situar un tiempoespacio en el que se dirime algo tan importante como el humanismo y los 
usos políticos y somáticos que se desprendan de ello allí mismo. Esta breve reflexión intenta problematizar por qué en algunos momentos de la analítica hecha sobre el experimento con humanos se ha apelado, eventualmente, al colonialismo. Ciertamente no de manera directa, pero sí a su actualización en los aparatos de adquisición y producción corporal en la historia presente en Guatemala. Siguiendo nuevamente a Chamayou, considero importante ponderar que "excepto si se ahonda en el idealismo del concepto, hay que considerar el humanismo como lo que es y fue; es decir, no como un sujeto unificado de la historia, sino como una arena de debate, una lengua común en la que se podían formular posiciones antagonistas" (2012: 53-54).

En lo que atiende a la problemática transhistórica es importante tener en cuenta el rescate que hace Chamayou sobre la advertencia de Hannah Arendt en contra de la idea de un "antisemitismo eterno"; es decir, aquello que puede convertirse en un estereotipo sin historia. Para Arendt, esta tesis, además de que no aporta nada, "lleva consigo un peligro político, a saber: la absolución de los autores de estas violencias al invocar una especie de trascendental de la historia europea" (Arendt en Chamayou, 2012: 149-150). Por otra parte, tengo en cuenta que para Arendt resultaba problemática "la teoría del chivo expiatorio". Es una "teoría de una violencia ejecutiva que elige a sus víctimas arbitrariamente (Ibídem)". En este contexto, resulta interesante pensar que el orden colonial habría que dislocarlo de un análisis transhistórico para comprenderlo específicamente a pesar de que sus técnicas puedan reactivarse en casos y momentos actuales con sus mismas técnicas. Mantener esta diferencia es importante para analizar los casos específicamente localizados y temporalizados.

Aunque hay que considerar que poco importa que estas prácticas de la experimentación hayan tenido lugar en un tiempo muy distinto al de la colonización, pues estas tecnologías de adquisición y producción corporal parecen originarse de allí. Es entonces que parece fundamental lo que afirma Mbembe al escribir que "la colonia representa el lugar en el que la soberanía consiste fundamentalmente en el ejercicio de un poder al margen de la ley (ab legibus solutus) donde la 'paz' suele tener el rostro de una 'guerra sin fin'” (2006: 37). 
La dotación de una humanidad a medias ha sido la condición para la primigenia subjetivación cristiana. Esa humanidad parece haber sido una utopía, algo que nunca se ha llegado a concretar para una indianiamalia. Un humanismo a medias -humanismo que no me genera nostalgias- parece haber sido un punto de atracción nunca alcanzado para los aspiracionistas posteriores.

Al tener en cuenta los campos semióticos y materiales en los que están implicados la salud y la degeneración, la seguridad y la defensa, y el provecho protésico de los cuerpos viles me recuerda lo que Haraway menciona respecto al discurso itinerante de la ciencia, el cual está "íntimamente implicado dentro de las otras grandes escrituras y lecturas colonizadoras y libertadoras tan fundamentales para las constituciones y disoluciones modernas de los cuerpos marcados por la raza, el sexo y la clase" (1995: 379-380). Al vincular este texto con la adquisición y producción de los cuerpos de experimentación, me parece que se debe tener en cuenta que el "miedo blanco" a la invasión de las bacterias sifilíticas ha hecho que, previo a tal invasión imaginaria y apocalíptica, los gestores de la salud pública estadounidense hayan capturado a los cuerpos anómalos como armas profilácticas en pro de su salud y bienestar.

Esta breve aproximación a la indianimalia permite pensar lo sucedido con aquellos inviables como humanos, correspondiendo con lo que sucedía con lxs indixs cuando se negaban a la servidumbre, teniendo como única opción esa condición ontológica, pues su condición de barbarie era un peligro para "la civilización". Me parece que se debe tener en cuenta que los cuerpos colonizados no fueron únicamente expuestos para los proyectos genocidas y los fines de mundos pasados y presentes. Un dispositivo distinto al exterminio y a los discursos y prácticas genocidas, más allá del peligro para la civilización, ha sido la conjunción de un aparato de producción corporal indianimal. Como tecnología colonial hay que notar que este se vincula mucho más a las prácticas de despojo permanente en correspondencia con el esclavismo.

Algunos historiadores han mencionado que la "humanización" de lxs indixs ha pertenecido al relato del fin del genocidio, no porque se conmisera con el sufrimiento humano de lxs indixs, sino porque respondía al interés de conservar la mano de obra. En este sentido, la restauración de la dignidad humana de lxs indixs colonizadxs fortaleció el derecho de los colonizadores 
sobre las tierras y la mano de obra en el Nuevo Mundo. Si se admite su humanidad a medias, no se puede negar su "éxito" para la explotación y el despojo. Se hace preciso interrumpir tal humanismo, aunque con ello se ha de abandonar nostálgicamente la pertenencia a tal especie.

Me planteo entonces, ¿Cómo se podría imaginar un horizonte indianimal fuera del marco de la inteligibilidad colonial, así quedemos fuera del mundo de lo normalmente inteligible? Me parece preciso pensar en la posibilidad de una insubordinación a la norma humana, imaginando proyectos de vida indianimales para la consecución de alianzas afecto-políticas, parentescos interespecies y corpodisidentes que erosionen al humanismo cristiano. Con la propuesta de una analítica del aparato de adquisición y de producción corporal quizás se pueda contribuir a imaginar algunas "lecciones" críticas para ensayar novedosos "gobiernos de si" que desarticulen los micro y macrofascismos contemporáneos de un humanismo cristiano colonial y nacional. Una alianza indianimal para carcomer las estrategias filantrópicas naturalizadas y naturalizantes. Con Haraway, es posible considerar que "los 'objetos', al igual que los cuerpos, no preexisten como tales. De manera similar, la 'naturaleza' no puede preexistir como tal, pero su existencia tampoco es ideológica. La naturaleza es un lugar común y una construcción discursiva poderosa, resultado de las interacciones entre actores semióticomateriales, humanos y no humanos" (Haraway, 1999: 124). Me parece preciso tener en cuenta que aún es posible inaugurar humanismos de figuraciones feministas multiespecies fuera de las biopolíticas de un humanismo colonial.

De aquí que quiero tener en cuenta las formulaciones de Gabriel Giorgi para las alianzas de lxs inapropiados/inapropiables. Animalidad e indianidad como alianza de filiación política contra las necropolíticas coloniales. Giorgi formula en su aproximación animalia queer:

[...] una "alianza biopolítica" contra la legibilidad normativa de lo humano, contra sus pedagogías culturales, contra sus ontologías políticas; se trata de pensar cómo leer esa alianza a modo que no constituya una sumatoria de "diferencias" ("animal", "queer") sino que, al contrario, ilumine epistemologías y políticas alternativas de lo viviente en las que lo que está en juego es una reinvención de un común de y entre los cuerpos. Esa posibilidad es, creo, la que 
se abre desde la animalia queer de la cultura: un campo de saberes alternativos sobre el cuerpo y una política de lo común (2013: 5).

En este sentido, me parece crucial una puesta en duda de los valores de un humanismo cristiano fuera del plano puramente moral. No se trata de delinear un horizonte incluyente dentro de la norma de lo humano, puesto que la dotación del alma, es decir, de la humanidad, para lxs inapropiadxs ha sido otorgada desde una biopolítica de la población y de la especie antropocéntrica.

Se puede argüir que el indio, al igual que el animal, ha sido una matriz de alteridad, en palabras de Giorgi. La animalización y la indianización han sido gestiones técnicas, fundaciones de jerarquización y diferenciación aprovechables para las políticas del buen cristiano, es decir, el humano colonialista. Me parece sugestivo para una analítica india lo que Giorgi arguye sobre el animal: el "animal funcionó como el otro constitutivo e 'inmediato' del humanismo mismo: toda distinción jerárquica entre clases, razas, géneros, sexualidad, etc.; todo antagonismo social o político; toda censura biopolítica entre cuerpos, pasa, casi invariablemente, por el animal” (2013: 9). La producción corporal del cuerpo colonizado diferenciado puede ser leído en vecindad con la gestión biopolítica del animal en los dispositivos de la experimentación médica y farmacológica. Es así que me parece que la producción del cuerpo sifilítico ha reciclado las tecnologías propias del humanismo cristiano con sus diferenciaciones jerárquicas animalizantes e indianizantes. 


\section{Fuente principal de investigación}

Records of Dr. John C. Cutler. Fondo archivístico alojado en: https:// www.archives.gov/research/health/cdc-cutler-records

\section{Bibliografía}

Agamben, Giorgio, (2010) Homo Sacer. El poder soberano y la nuda vida. Valencia: Pre-Textos.

Archivo General de Centroamérica, Dirección de los Archivos de la Paz y Archivo Histórico de la Policía Nacional -Guatemala- (2011) Investigación archivistica sobre experimentos practicados en seres humanos en Guatemala, 1947-1948. Guatemala.

Bianchi, Eugenia (2019) "Ciencias sociales, salud mental y control social. Notas para una contribución a la investigación”, Salud Mental y Comunidad. 6 (7), Ediciones UNLa, Remedios de Escalada, pp. 1228. Disponible en: http://www.unla.edu.ar/documentos/centros/ salud_mental_comunitaria/revista/saludmentalycomunidad7.pdf

Bianchi, Eugenia (2018) "Saberes, fármacos y diagnósticos. Un panorama sobre producciones recientes en torno a la farmacologización de la sociedad", Psicología, Conocimiento y Sociedad. 8(2), pp. 214-257. Disponible en: http://www.scielo.edu.uy/pdf/pcs/v8n2/1688-7026pcs-8-02-147.pdf

Centers for Disease Control and Prevention (CDC) Report on Finding from the U. S. (2010) Public Health Sevice Sexually Transmitted Disease Inoculation Study of 1946-1948, Based on Review of Archived Papers of John Cutler, MD. University of Pittsburgh. Disponible en: https:// stacks.cdc.gov/view/cdc/5797

Comisión Presidencial para el Esclarecimiento de los Experimentos Practicados con Humanos en Guatemala (2011) Consentir el daño. Experimentos médicos de Estados Unidos en Guatemala, 1946-1948. Guatemala.

Comisión Presidencial para el Esclarecimiento de los Experimentos en humanos en Guatemala (2011) Experimentos en seres humanos el caso Guatemala 1946- 48, Guatemala. Disponible en: https://www.paho.org/gut/ index.php?option=com_docman\&view=download\&category_ 
slug=1-gerencia-y-coordinacion\&alias=439-informe-de-la-comisioninvestigadora-experimentos-1946-1948-gobierno-de-guatemalanoviembre-2011\&Itemid $=518$

Chamayou, Grégoire (2012) Las cazas del hombre. El ser humano como presa de la Grecia de Aristóteles a la Italia de Berlusconi. Madrid: errata naturae.

Chamayou, Grégoire (2008) Les corps vils. Expérimenter sur les êtres humains aux XVIIIe et XIXe siècles. Paris: Éditions La Découverte.

Chivalán, Marco (2019) “Prostitución como 'mal necesario' a finales del XIX en la Ciudad de Guatemala: sexopolítica, pornotopía y supremacía masculina" en Producción corporal. Interfiriendo engranajes de violencia en Guatemala. Guatemala: Asociación para el Avance de las Ciencias Sociales en Guatemala.

De Lauretis, Teresa (2000) Diferencias. Etapas de un camino a través del feminismo. Madrid: horas y HORAS.

Dorlin, Elsa (2009) La matrice de la race. Généalogie sexuelle et coloniale de la Nation française. Paris: Éditions La Découverte.

Foucault, Michel (2005) El poder psiquiátrico. Buenos Aires: Fondo de Cultura Económica.

Foucault, Michel (1995) Historia de la sexualidad. 1. La voluntad de saber. Madrid: Siglo veintiuno de España editores.

Foucault, Michel (1990) Tecnologías del yo y otros textos afines. Barcelona: Paidós, Institut de Ciències de l'Educació-Universitat Autònoma de Barcelona.

Giorgi, Gabriel (2013) "La lección animal: pedagogías queer” en Domínguez, Nora (ed.), Cuerpo y escritura. El género como pregunta. Centro de Estudios de Teoría y Crítica Literaria, CETYCLI, Rosario. Boletín No. 17, pp. 1-40. Disponible en: https://www.cetycli.org/cboletines/ ce93e200c3-gabriel_giorgi17.pdf

Haraway, Donna (1995) Ciencia, cyborgs y mujeres. La reinvención de la naturaleza. Madrid: Ediciones cátedra, Universitat de València, Instituto de la Mujer.

Haraway, Donna (1999) "Las promesas de los monstruos: Una política para otros inapropiados/bles", en Politica y Sociedad. Vol. 30 (1999) pp. 121-163. Disponible en: https://www.relmecs.fahce.unlp.edu.ar/ article/view/Relmecse052/10737 
Haraway, Donna (2016) Manifiesto de las especies compañeras. Bilbao: Sans Soleil Ediciones.

Haraway, Donna(2004a)Testigo_Modesto@Segundo_Milenio.HombreHembra@_ Conoce_Oncoratón(R). Feminismo y tecnociencia. Barcelona: Editorial de la Universitat Oberta de Catalunya.

Haraway, Donna (2004b) The Haraway Reader. New York y Londres: Routledge.

Mbembe, Achille (2006) Necropolitica. Santa Cruz de Tenerife: Editorial Melusina.

Murillo, Susana (2008) Colonizar el dolor. La interpelación ideológica del Banco Mundial en América Latina. El caso argentino desde Blumberg a Cromañón. Buenos Aires: Clacso Libros. Disponible en: https:// www.clacso.org.ar/libreria-latinoamericana/buscar_libro_detalle. php? $c$ ampo=titulo\&texto $=\&$ id_libro $=60$

Murillo, Susana (2018) "El gobierno de las infancias" en Faraone, Silvia y Eugenia Bianchi, (comp.) Medicalización, salud mental e infancias. Perspectivas y debates desde las ciencias sociales en Argentina y el sur de América Latina. Buenos Aires: Ed. Teseo. Disponible en: https://www. teseopress.com/medicalizacion/chapter/el-gobierno-de-las-infancias/

Murillo, Susana (2015a) "La administración de la vida a través de la muerte. De la medicina clínica a la biomedicina” en Faraone, Silvia, Bianchi, Eugenia y Soraya Giraldez (comp.), Determinantes de la salud mental en Ciencias Sociales. Actores, conceptualizaciones, politicas y prácticas en el marco de la ley 26,657. Buenos Aires: Editorial UBA Sociales.

Murillo, Susana (2015b) Neoliberalismo y gobiernos de la vida. Diagrama global y sus configuraciones en la Argentina y América Latina. Buenos Aires: Biblos.

Preciado, Beatriz (2012) Somateca. Producción biopolitica, feminismos, prácticas queer y trans. Seminario dirigido por Beatriz Preciado. Madrid: Museo Nacional Centro de Arte Reina Sofía. Digital disponible en: https:// www.museoreinasofia.es/pedagogias/centro-de-estudios/somatecaproduccion-biopolitica-feminismos-practicas-queer-trans.

Preciado, Beatriz (2008) Testo Yonqui. Madrid: Espasa.

Presidential Commission for the Study of Bioethical Issues, (2011) "Eticamente imposible". Investigación sobre las STD en Guatemala desde 1946 hasta 
1948. Washington, D. C. Disponible en: https://bioethicsarchive. georgetown.edu/pcsbi/sites/default/files/EticamenteImposible_ Spanish_Translation.pdf

Reverby, Susan, (2011) “'Normal Exposure' and Inoculation Syphilis: A PHS 'Tuskegee' Doctor in Guatemala, 1946-1948” en The Journal of Policy History. 23 (1) pp. 7-28. Doi: 10.1017/S0898030610000291

Reverby, Susan, (2013) Examining Tuskegee: The Infamous Syphilis Study and its Legacy (The John Hope Franklin Series in African American History and Culture). Chapel Hill, NC: The University of North Carolina Press. 